\title{
The hierarchy of multiple many-body interaction scales in high-temperature superconductors
}

\author{
W. Meevasana, , , X.J. Zhou, ${ }^{1,2}$ S. Sahrakorpi, ${ }^{3}$ W.S. Lee, ${ }^{1}$ W.L. Yang, ${ }^{1,2}$ K. Tanaka,,${ }^{1,2}$ N. Mannella, ${ }^{1,2}$ T. \\ Yoshida, ${ }^{1,4}$ D. H. Lu, ${ }^{1}$ Y.L. Chen, ${ }^{1}$ R.H. He ${ }^{1}$ Hsin Lin,${ }^{3}$ S. Komiya, ${ }^{5}$ Y. Ando, ${ }^{5}$ F. Zhou, ${ }^{6}$ W.X. Ti, ${ }^{6}$ J.W. \\ Xiong, ${ }^{6}$ Z. X. Zhao,${ }^{6}$ T. Sasagawa,,${ }^{1,7}$ T. Kakeshita,${ }^{7}$ K. Fujita,${ }^{7}$ S. Uchida,${ }^{7}$ H. Eisaki, ${ }^{8}$ A. Fujimori ${ }^{4}{ }^{2}$.

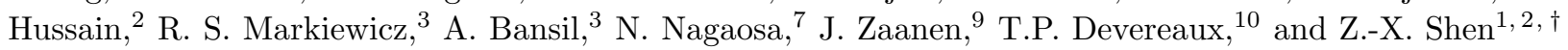 \\ ${ }^{1}$ Department of Physics, Applied Physics, and Stanford Synchrotron \\ Radiation Laboratory, Stanford University, Stanford, CA 94305 \\ ${ }^{2}$ Advanced Light Source, Lawrence Berkeley National Lab, Berkeley, CA 94720 \\ ${ }^{3}$ Physics Department, Northeastern University, Boston, MA 02115 \\ ${ }^{4}$ Department of Complexity Science and Engineering, University of Tokyo, Kashiwa, Chiba, Japan \\ ${ }^{5}$ Central Research Institute of Electric Power Industry, Iwato-kita, Komae, Tokyo, Japan \\ ${ }^{6}$ National Lab for Superconductivity, Institute of Physics, Chinese Academy of Sciences, Beijing, China \\ ${ }^{7}$ CREST, Department of Applied Physics, Advanced Materials Science, \\ and Superconductivity, University of Tokyo, Bunkyo-ku, Tokyo, Japan \\ ${ }^{8}$ Nanoelectronic Research Institute, AIST, Tsukuba 305-0032, Japan \\ ${ }^{9}$ The Instituut-Lorentz for Therorectical Physics, Leiden University, Leiden, The Netherlands \\ ${ }^{10}$ Department of Physics, University of Waterloo, Waterloo, Ontario, Canada N2L 3G1
}

(Dated: November 30, 2006)

\begin{abstract}
To date, angle-resolved photoemission spectroscopy has been successful in identifying energy scales of the many-body interactions in correlated materials, focused on binding energies of up to a few hundred meV below the Fermi energy. Here, at higher energy scale, we present improved experimental data from four families of high- $T_{c}$ superconductors over a wide doping range that reveal a hierarchy of many-body interaction scales focused on: the low energy anomaly ("kink") of $0.03-0.09 \mathrm{eV}$, a high energy anomaly of $0.3-0.5 \mathrm{eV}$, and an anomalous enhancement of the width of the LDA-based $\mathrm{CuO}_{2}$ band extending to energies of $\approx 2 \mathrm{eV}$. Besides their universal behavior over the families, we find that all of these three dispersion anomalies also show clear doping dependence over the doping range presented.
\end{abstract}

\section{INTRODUCTION}

Many-body interaction is a key to understanding novel properties of quantum matter. As an extreme example, the complexity due to charge, spin, and lattice interactions in high- $T_{c}$ superconductors makes it difficult to identify the essential microscopic ingredients for the basic model - a reason behind the current debate on the mechanism. The energy-momentum dispersion relationship measured by angle-resolved photoemission spectroscopy (ARPES) provides an excellent tool for identifying these scales. Energy scales where these interactions are manifest usually provide important insights into the nature of the interactions. At an early stage, APRES proved to be successful in identifying the energy scale of the $\mathrm{d}$-wave gap in high- $\mathrm{T}_{c}$ superconductors ${ }^{1,2}$. To date, a focus of the discussion in ARPES has been on the nature of electron-boson coupling, which manifests itself in the form of the low energy anomaly ("kink") near 0.03$0.09 \mathrm{eV}^{2,3,4,5,6,7,8,9,10}$. However, little attention has been paid to the features at higher binding energies.

Given the strong many-body interactions and complex band structure, one expects only incoherent features and complex spectral weight modulation at high energy. The fact that one can see neatly defined momentum dependent features which are robust against doping and measuring conditions (e.g. photon energy) is unexpected and thus provides a new oppor- tunity to understand many-body effects beyond what have traditionally been the points of focus by ARPES, other than some early work on oxygen $p$ bands 11,12 . Here, we report ARPES experiments on four families of high- $T_{c}$ cuprates over a wide range of dopings: $\mathrm{Bi}_{2} \mathrm{Sr}_{2} \mathrm{CuO}_{6}(\mathrm{Bi} 2201), \mathrm{Bi}_{2} \mathrm{Sr}_{2} \mathrm{CaCu}_{2} \mathrm{O}_{8}$ ( $\mathrm{Bi} 2212$ ), $\mathrm{Ba}_{2} \mathrm{Ca}_{3} \mathrm{Cu}_{4} \mathrm{O}_{8}\left(\mathrm{O}_{\delta}, \mathrm{F}_{1-\delta}\right)_{2}$ (F0234), and $\mathrm{La}_{2-x} \mathrm{Sr}_{x} \mathrm{CuO}_{4}$ (LSCO). All of their measured energy-momentum dispersion relationships reveal the simultaneous presence of three energy scales, marked as 1-3 in Fig. 1d: 1) the band bottom, at nearly $2 \mathrm{eV}$ in optimally-doped Bi2201, which is deeper than predicted by LDA calculation, 2) the high-energy anomaly (HEA) at $\approx 0.3-0.5 \mathrm{eV}$ (green arrow) and 3) the low-energy "kink" (LEK) near $70 \mathrm{meV}$ (red arrow) which can be better seen in an enlarged energy window. Various aspects of these energy scales, including the peculiar doping dependent effects, will be discussed to obtain insights on many-body interactions and their interplay in cuprates.

\section{EXPERIMENT AND LDA CALCULATION}

We have measured four families of high- $T_{c}$ cuprates: $\mathrm{Bi}_{2} \mathrm{Sr}_{2} \mathrm{CuO}_{6} \quad(\mathrm{Bi} 2201), \quad \mathrm{Bi}_{2} \mathrm{Sr}_{2} \mathrm{CaCu}_{2} \mathrm{O}_{8} \quad$ (Bi2212), $\mathrm{Ba}_{2} \mathrm{Ca}_{3} \mathrm{Cu}_{4} \mathrm{O}_{8}\left(\mathrm{O}_{\delta}, \mathrm{F}_{1-\delta}\right)_{2}(\mathrm{~F} 0234) \stackrel{14}{=}$, and $\mathrm{La}_{2-x} \mathrm{Sr}_{x} \mathrm{CuO}_{4}$ (LSCO). The Bi2201 samples are optimally-doped of $\mathrm{Tc}=35 \mathrm{~K}$ and non-superconducting overdoped. The 

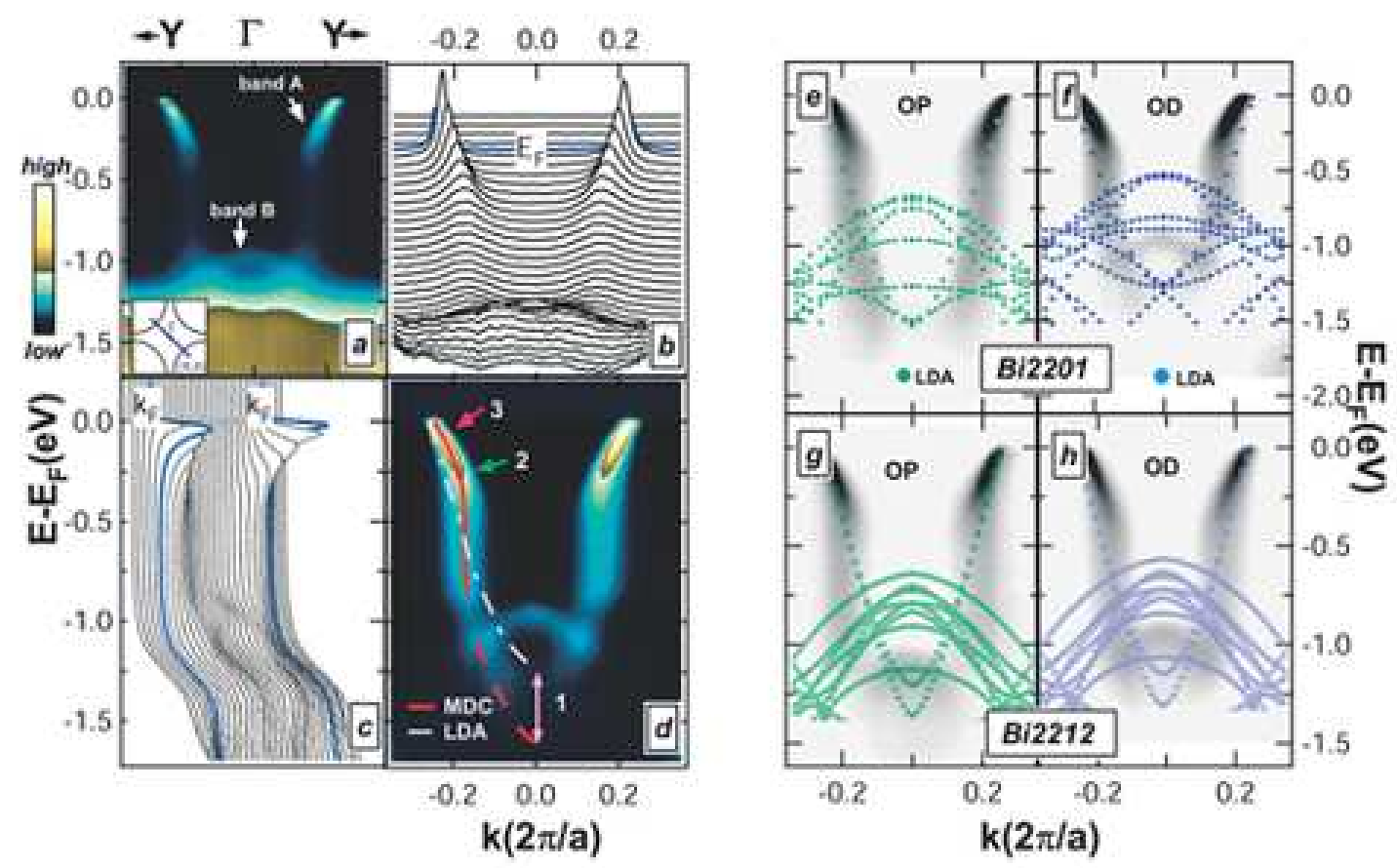

FIG. 1: (a) Raw ARPES spectrum, along $(0,0)$ to $(\pi, \pi)$ direction, of non-superconducting overdoped Bi2201 at $\mathrm{T}=30 \mathrm{~K}$ while (b) and (c) represent its MDCs and EDCs respectively. (d) the raw data is normalized with angle-integrated EDC profile for clearer view and the numbers 1-3 mark the wider band width, the high and low-energy anomalies respectively. Normalized ARPES spectra are compared with LDA calculations as follows: (e) OP Bi2201 with $\mathrm{T}_{c}=35 \mathrm{~K}$ (T=45K), (f) OD Bi2201, non-superconducting $(\mathrm{T}=30 \mathrm{~K}),(\mathrm{g}) \mathrm{OP}$ Bi2212 with $\mathrm{T}_{c}=96 \mathrm{~K}\left(\mathrm{~T}=110 \mathrm{~K}\right.$, LDA from Ref 13 ) and (h) OD Bi2212 with $\mathrm{T}_{c}=$ $65 \mathrm{~K}(\mathrm{~T}=76 \mathrm{~K})$. Inset in Fig. 1a shows the momentum space of the data. Note that the LDA bands in panel (h) are obtained by rigidly shifting the bands in $(\mathrm{g})$ to account for the correct doping level. It is not clear if feature B should be matched to the top of the band structure in (e) or (f) at $\Gamma$; however, this uncertainty is not important for our argument which only requires a relative shift in going from the OP to the OD case.

Bi2212 samples are optimally-doped of $\mathrm{Tc}=92 \mathrm{~K}$ and overdoped of $\mathrm{Tc}=65 \mathrm{~K}$. The F0234 samples are of Tc $=60 \mathrm{~K}$. And, the LSCO samples has a wide range of dopings: $\mathrm{x}=0.03,0.05,0.063,0.07,0.075,0.09,0.12$, $0.15,0.22$ and 0.3 . The measurements were carried out on beamline 10.0.1 at the ALS, using a Scienta R4000 electron energy analyzer. This analyzer has the advantage of a large-angle window which can cover the band dispersion across the Brillouin zone as shown in Fig. 1. We stress that the wide angle scan allowed us to record above data without resorting to manual symmetrization. The photon energies are $37,40,41, \ldots, 45$ and $55 \mathrm{eV}$. The energy resolution between 12 and 20 $\mathrm{meV}$ was used for various measurements on different samples, and the angular resolution is 0.3 degree. The samples were cleaved in situ in vacuum with a base pressure better than $4 \times 10^{-11}$ torr. The samples were measured both in normal and superconducting states.

LDA results here are based on full-potential wellconverged computations for the appropriate lattice structures, described in greater details in Ref. $\frac{13}{}$ and ${ }^{15}$.

\section{RESULTS}

Fig. 1a shows the raw ARPES image of the strongly overdoped Bi2201 sample while its raw momentumdistribution-curves (MDC) and energy-distributioncurves (EDC) are shown in Fig. 1b and c, respectively. To see the band near the bottom more clearly, the raw ARPES image is divided by its profile of angle-integrated EDC 16 , as shown in Fig. 1d; this procedure will not change the MDC-peak position at any given energy. We also note that this renormalization procedure which is used in Fig. 1d-h and Fig.2 is only for the purpose of displaying the lower and higher energy features together since otherwise the intensity at the higher-energy region will be too high to have a reasonable displaying contrast. The raw data without this renormalization procedure can be seen in Fig. 1a-c and Fig. 6a-i.

As marked by numbers 1-3 in Fig. 1d, we will focus on how our new data reveal the simultaneous presence of three energy scales as follows. 


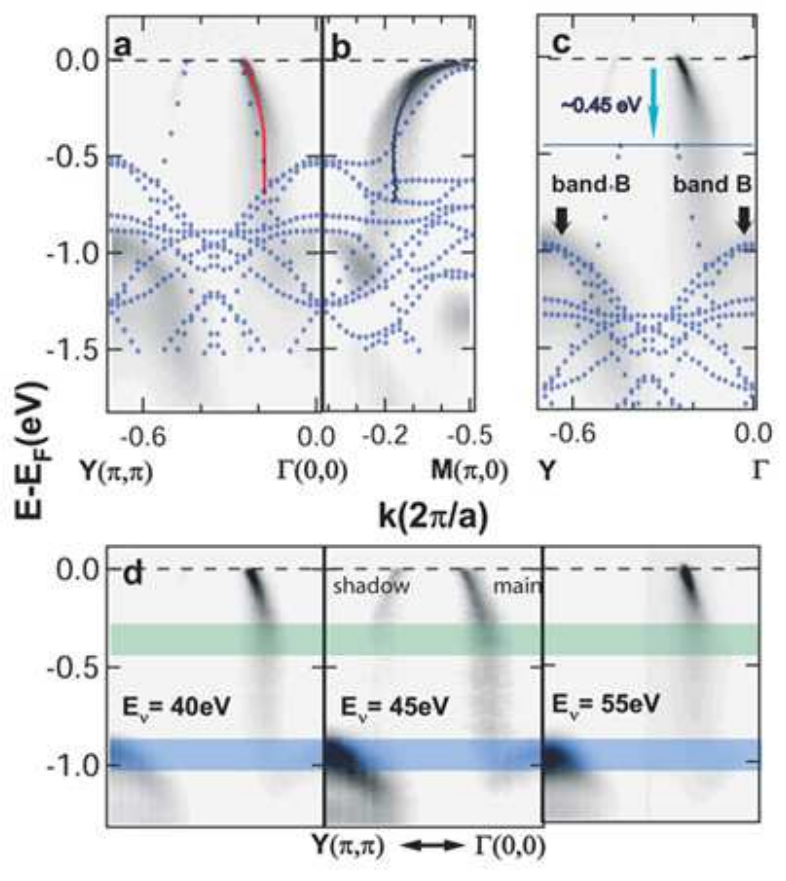

FIG. 2: The comparison of normalized ARPES spectra and LDA calculations along (a) $(\pi, \pi)$ to $(0,0)$ or nodal direction and (b) $(0,0)$ to $(\pi, 0)$ or antinodal direction in OD nonsuperconducting Bi2201 system. (c) shows the agreement of ARPES spectrum and LDA calculation of high-energy band when shifting the LDA by $450 \mathrm{meV}$ to the higher energy. (d) The comparison of the spectra at photon energies, $\mathrm{E}_{\nu}=40,45$ and $55 \mathrm{eV}$ where the green shaded region denotes the energy scale of HEA and blue shaded region indicates the top of the band $\mathrm{B}$. We note that for $\mathrm{E}_{\nu}=55 \mathrm{eV}$, due to the matrix element effect, the intensity of the left band B is large and therefore we have adjusted the color scale so that we can see the energy scales of both band A and B clearly.

\section{A. anomalous enhancement of the LDA-based $\mathrm{CuO}_{2}$ band width}

As shown in Fig. 1e-1h, ARPES spectra of optimallydoped (OP) and overdoped (OD) samples of Bi2201 and Bi2212 systems are overlaid on the corresponding LDA calculations. As seen in all measured samples, the first peculiar feature, especially at lower doping, is that the ARPES band width is found to be wider than LDA calculation. This is anomalous as one expects interactions to enhance the mass and reduce the band width. By extrapolating the band (e.g. the red dashed line in Fig. 1d), one can get an estimate of the band width that is suitable for qualitative discussion. With doping, the discrepancy between the band widths obtained from ARPES and LDA seems to be reduced.

Additional evidence that these high energy dispersions still contain useful information comes from band B in Fig. 1a which shows a maximum at $\Gamma$ point near $1 \mathrm{eV}$. This band has a correspondence to an LDA band and thus

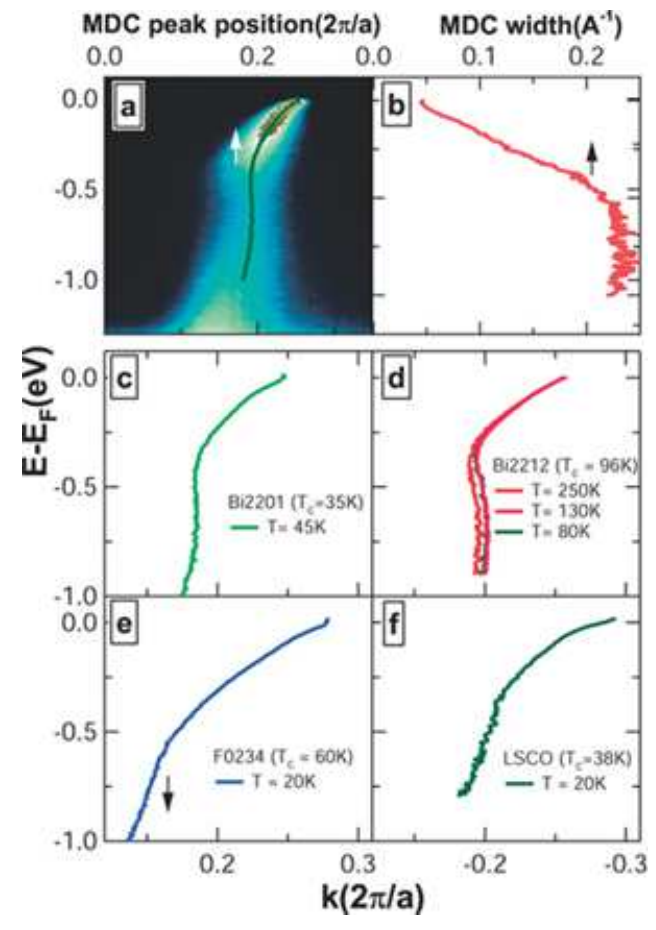

FIG. 3: (a) shows MDC-peak dispersion plotted on top of ARPES spectrum and (b) shows corresponding MDC width in OP Bi2201 system $\left(\mathrm{T}_{c}=35 \mathrm{~K}\right)$. (c)-(f) show the MDCderived dispersions of $\mathrm{Bi} 2201(\mathrm{Tc}=35 \mathrm{~K}), \mathrm{Bi} 2212(\mathrm{Tc}=65 \mathrm{~K})$, $\mathrm{LSCO}(\mathrm{Tc}=38 \mathrm{~K})$ and $\mathrm{F} 0234(\mathrm{Tc}=60 \mathrm{~K})$ respectively. Additionally, the temperature dependence of Bi2212 and LSCO dispersion is shown in (d) and (f).

provides confidence in the data at higher energy scales, which has been largely unexplored in the cuprates. From the LDA calculation with orthorhombic distortion, this band is the band at $\mathrm{Y}$ point (left arrow, Fig. 2c), which is folded around the $(\pi / 2, \pi / 2)$ point. We then compare the LDA and ARPES top part of this band $B$ at $\Gamma$ point by shifting the LDA band down. A good agreement of ARPES and LDA of this concave-down band (see Fig. 2 c) can be obtained if the LDA is shifted down $\approx 0.45$ $\mathrm{eV}$ for OD Bi2201 sample and the shifted energy increases to $\approx 0.8 \mathrm{eV}$ for OP Bi2201 sample $\frac{17}{}$, leading to a filled band width near $2 \mathrm{eV}$. A similar behavior is also observed in the Bi2212 system ${ }^{17}$ (Fig. 1g-h). This band width enhancement was also seen earlier in undoped $\mathrm{Ca}_{2} \mathrm{CuO}_{2} \mathrm{Cl}_{2}$ (CCOC) as its high-energy dispersion matches with the LDA calculation shifted by $0.7 \mathrm{eV} \stackrel{18}{ }$.

\section{B. high energy anomaly of 0.3-0.5 eV}

Next, we discuss the high energy anomaly (HEA) near $0.3-0.5 \mathrm{eV}$. We extract the MDC peak position by fitting to Lorentzian curves, as shown by the red and blue curves in Fig. 2a and 2b. We note that especially at high-binding energy, MDC peak position may not rep- 


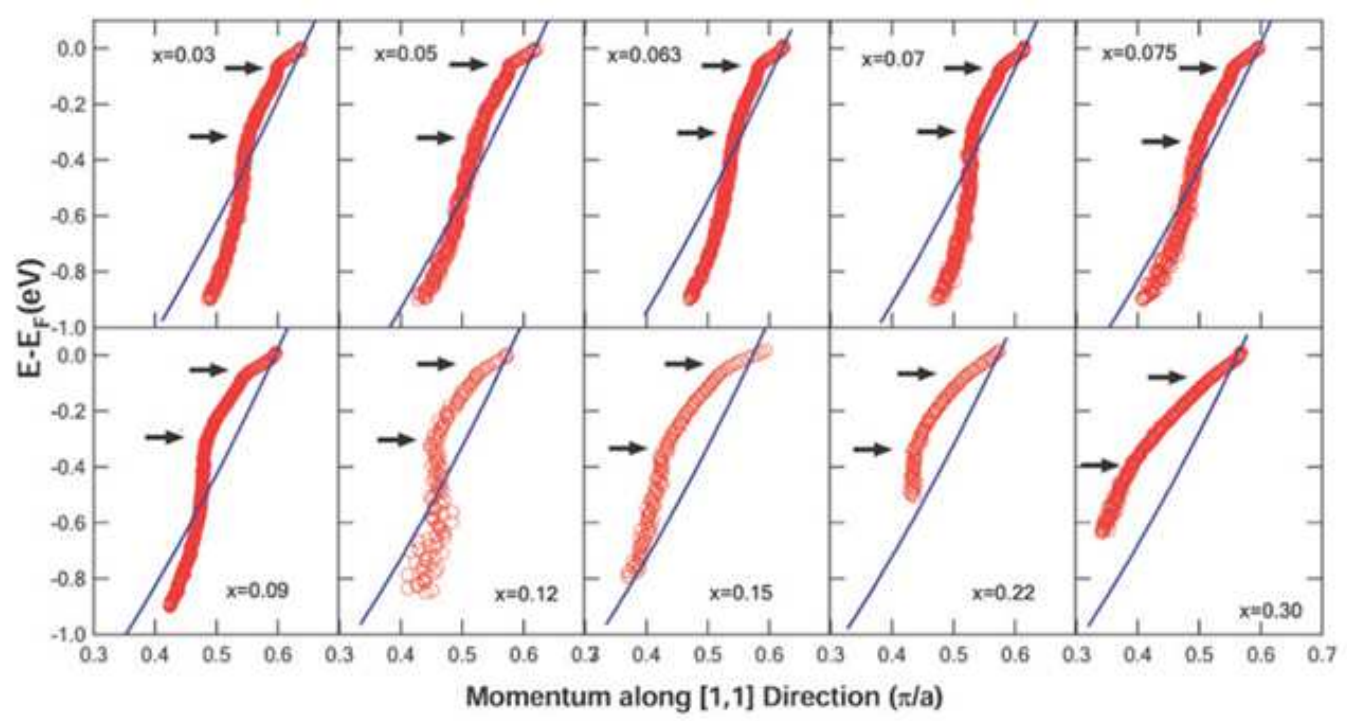

FIG. 4: The doping dependence of high-energy dispersion (red circles) in LSCO system at T $=20 \mathrm{~K}$ at doping $\mathrm{x}=0.03$ to 0.30 . LDA curves for various dopings (blue lines) are obtained by appropriate rigid shifts of the computations for $\mathrm{x}=0$.

resent the real dispersion. Explained in appendix, a full $2 \mathrm{D}$ analysis, which directly extracts the spectral function, $A(k, \omega)$ and matrix element term separately at once, can avoid the problem of the MDC or EDC analysis alone. However, since the kink-like structure is so large, the MDC-derived dispersion should be able to approximately identify the energy scale of the HEA. To check the 3 dimensional behavior of the band $\mathrm{A}$ and $\mathrm{B}$, we perform the measurements at various photon energies $40,41, \ldots, 45$ $\mathrm{eV}$ and $55 \mathrm{eV}$, probing different perpendicular momenta $k_{z}$. As shown in Figs. 2d, we find that HEA scale and the top of Band B are not very sensitive to the photo energies while as well these energy scales in our LDA calculations do not show strong $k_{z}$ dependence.

This HEA is present in various cuprate families. While earlier seen in undoped CCOC $\stackrel{18}{ }$, the MDC-peak-position graphs of Bi2201, Bi2212, LSCO and F0234 plotted in Fig. 3, reveal its universality. The energy scales are around 0.3-0.4 eV in Bi2201, Bi2212 and LSCO while around 0.5-0.6 eV in F0234 (for electron-doped band). HEA persists in both superconducting and nonsuperconducting samples, albeit its strength depends on doping. Fig. 4 shows the plots of MDC-peak position of LSCO samples which cover a wide doping range, $\mathrm{x}=0.03$ to 0.30 . From the figure, the HEA energy does not change much with doping. However, if we define the size of HEA to be the difference between the MDC-derived and LDA dispersion, it increases upon doping in this range. Similar doping-dependent behavior is also observed in Bi2201 and Bi2212 samples which cover a narrower range of doping. For superconducting samples, the HEA persists above and below Tc. consistent with ARPES data, from in-plane optical conductivity of Bi2212 29 , Norman and Chubukov recently report that the real part of the self-energy is large with a maximum value around 0.3-0.4
$\mathrm{eV}^{20}$.

\section{C. low energy "kink" of 0.03-0.09eV}

Finally, the low energy "kink" (LEK) around 0.03-0.09 $\mathrm{eV}$ is indicated with arrows in Fig. 2d, and upper arrows in Fig. 4 and Fig. 5a. Since this feature has been already discussed with regard to the interaction of electron to

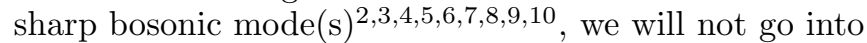
the details of this feature, except commenting upon its interesting doping dependence. For LEK, the size of this feature, which is interpreted as strength of electron-boson coupling, reduces upon doping in $\mathrm{LSCO}^{9}$ and $\mathrm{Bi}^{2} 201^{10}$ while the size of HEA defined previously increases upon doping. It is then intriguing to ask whether an interplay of these two scales of low and high-energy anomalies will affect our understanding of the doping dependent effects seen in cuprates.

\section{DISCUSSION}

The presence of three energy scales in the same data set hints at the hierarchy of interactions that are important to the dynamics of electrons in cuprates. Aside from LEK which we believe is caused by electron-phonon interaction $6.8,9,10$, HEA and the expanded band width are new observations that require more discussion.

To gain more insights into the nature of the energy scales observed, Fig. 5 compares the data of the superconducting (SC) sample, OP Bi2201, with that of the antiferromagnetic (AF) parent compound $\mathrm{CCOC}$ and ferromagnetic (FM) $\mathrm{La}_{1.2} \mathrm{Sr}_{1.8} \mathrm{Mn}_{2} \mathrm{O}_{7}$ (LSMO). Comparison of LSMO and cuprates may give us some insight since 


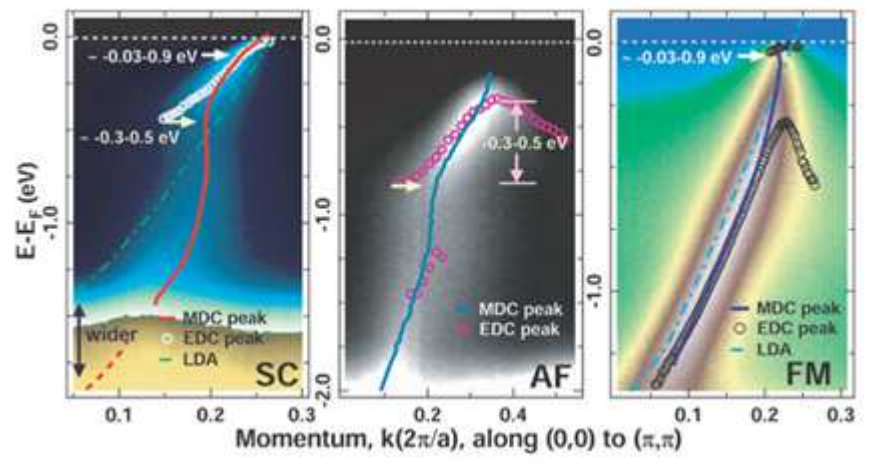

FIG. 5: Comparison of electronic structures of three perovskites in (a) superconducting (SC), (b) antiferromagnetic (AF) and (c) ferromagnetic (FM) phases. The images show ARPES data of (a) optimally-doped Bi2201, (b) undoped CCOC, and (c) LSMO.

LSMO is reported to have similar pseudogap behavior 21 . The LSMO comparison yields two insights: i) HEA is probably related to antiferromagnetism as it is absent in the ferromagnetic state and ii) the LDA calculation apears to give a correct band width if antiferromagnetism is not present. As for LEK near $0.03-0.09 \mathrm{eV}$, it is seen in metallic cuprates and LSMO but not in insulating CCOC where the polaron effect is too strong, thus suppressing the quasiparticle weight dramatically 22 .

HEA may be related to the short range Coulomb interaction as in the Hubbard (or t-J) model. Calculations using these models show that in undoped $\mathrm{Sr}_{2} \mathrm{CuO}_{2} \mathrm{Cl}_{2}$, the quasiparticle band width of the part below HEA is set by the $\mathrm{J}$ scale to be around $2-3 \mathrm{~J} \simeq 0.25-0.35 \mathrm{eV} 23$ while the higher energy part is presumed to be the incoherent band with t scale $\frac{18}{}$. HEA in the Hubbard calculation, which may come from the meeting of the quasiparticle band and the incoherent lower-Hubbard band, can be seen from the slightly underdoped to overdoped regime ${ }^{24}$; however, a further check still remains in the small doping regime where the calculation is challenging. In $\mathrm{SrVO}_{3}$, a feature similar to HEA is seen in ARPES 25 and in LDA+DMFT calculation ${ }^{26}$. With these models, the size of HEA is expected to be less pronounced upon doping $^{27}$ which is opposite to the doping effect we see in Fig. 4.

One possible way to understand the anomalous doping dependence could be the interplay between electronelectron and electron-phonon interactions. This drives the system into the polaronic regime in the underdoped samples $\frac{22}{2}$, making a quantitative analysis of the size of the HEA difficult because obtaining polaronic physics correctly via an MDC analysis is challenging. Since the polaron physics is very strong in lightly-doped regime, this may artificially suppress the HEA size.

Another possibility is that HEA comes from the renormalization effect by bosonic mode(s). The in-plane plasmon and the two-magnon mode are two possible can- didates. The coupling strength of in-plane plasmon increases upon doping, as shown by the energy-loss function in Ref. $\frac{28}{2}$. Qualitatively, the in-plane plasmon should give a very similar doping effect to that shown in Fig. 4. The problem is that the plasmon mode energy in LSCO is around $0.8 \mathrm{eV}$, or twice as large. On the other hand, the two-magnon mode energy is right in the window for the hole-doped cuprates $(0.3-0.4 \mathrm{eV})$ but not for FO234, electron-doped band ( $0.5-0.6 \mathrm{eV})$ while its strength quickly reduces upon doping 29 .

Given the above alternative interpretations and issues related to them, despite the quantitative problem of the HEA size as a function of doping, the anomalous energy scale is still likely caused by the Mott-Hubbard physics. The fact that HEA is absent in FM LSMO (Fig. 5) but present in the Mott-Hubbard system $\mathrm{SrVO}_{3}$ suggests that it is related to antiferromagnetism and MottHubbard physics.

Next is the enhancement of LDA band width which is anomalous because interactions usually reduce the band width. One possible candidate here could be the poor screening effect. With the Hartree-Fock equation of free electron, the Coulomb exchange term without the screening effect ${ }^{30}$ will give rise to a negative term which results in an increased band width; the effect of poor screening can be seen in semiconductors. In cuprates, the electrons in lightly-doped systems are poorly screened compared to the overdoped regime and hence the discrepancy of band width is larger in underdoped systems. We note here that, for the $\mathrm{SrVO}_{3}$ system, LDA+DMFT calculation ${ }^{26}$ gives the band width correctly. This distinction between $\mathrm{SrVO}_{3}$ and cuprates should be investigated further.

\section{CONCLUSION}

In conclusion, based on data from four families of cuprates over a wide doping range, we present evidence of a hierarchy of multiple energy scales in cuprates focused on: the low energy anomaly of $0.03-0.09 \mathrm{eV}$, a high energy anomaly of $0.3-0.5 \mathrm{eV}$, and an anomalous enhancement of LDA band width, extending over an energy scale of $\approx$ $2 \mathrm{eV}$. These results suggest that electron-phonon interaction, short-range Coulomb interaction and poor screening should be all considered to understand the nature of cuprates.

\section{Acknowledgments}

We thank A. Macridin, B. Moritz, M. Jarrell, K.M. Shen, D. Scalapino, D. van der Marel, N.J.C Ingle, F. Baumberger for helpful discussions. The work at SSRL and ALS are supported by DOE's Office of Basic Energy Sciences under Contracts No. DE-AC02-76SF00515 and DE-AC03-76SF00098. The work at Stanford is supported by NSF DMR-0304981 and ONR N00014-04-10048. W.M. acknowledges DPST for the financial sup- 
port. The work at Northeastern is supported by the US DOE contract DE-AC03-76SF00098 and benefited from the allocation of supercomputer time at NERSC and Northeastern's Advanced Scientific Computation Center (ASCC). The work at IOP-CAS is supported by NSF of China. T.P.D. acknowledges ONR N00014-05-1-0127.

\section{APPENDIX: A FULL 2D ANALYSIS}

In the following, we will show that interestingly, a 2D analysis could describe ARPES data well, covering a large energy and momentum space, by a compact set of parameters. This is an attempt to go beyond the conventional EDC or MDC analysis alone. However, we note that the physical meaning of such a parameterization still remains to be explored.

Here we will apply the 2D analysis on the ARPES data of Pb-substituted Bi2201. The overdoped (OD) samples, $\mathrm{Pb}_{0.38} \mathrm{Bi}_{1.74} \mathrm{Sr}_{1.88} \mathrm{CuO}_{6+\delta}$, are non-superconducting $\left(\mathrm{T}_{\mathrm{c}}\right.$ $<4 \mathrm{~K})$. ARPES data were collected with a photon energy of $42 \mathrm{eV}$. The energy resolution was set to $18 \mathrm{meV}$. The linear polarization of the light source is fixed to be inplane along $(0,0)$ to $(\pi, \pi)$ throughout the measurement. Note that the fitted matrix element, which is shown in Fig. 6d-i, refers to this particular experimental geometry.

The intensity measured in an ARPES experiment on a $2 \mathrm{D}$ material here will be parameterized by ${ }^{2}$

$$
I(\mathbf{k}, \omega)=I_{0}(\mathbf{k}, \nu, \mathbf{A}) f(\omega) \mathcal{A}(\mathbf{k}, \omega)
$$

where $I_{0}(\mathbf{k}, \nu, \mathbf{A})$ is proportional to the one-electron matrix element and dependent on the polarization, momentum and energy of the incoming photon, $f(\omega)$ is the Fermi function. $\mathcal{A}(\mathbf{k}, \omega)$ is the single-particle spectral function given by

$$
\mathcal{A}(\mathbf{k}, \omega)=\frac{(-1 / \pi) \operatorname{Im} \Sigma(\mathbf{k}, \omega)}{\left[\omega-\epsilon_{k}^{0}-\operatorname{Re} \Sigma(\mathbf{k}, \omega)\right]^{2}+[\operatorname{Im} \Sigma(\mathbf{k}, \omega)]^{2}}
$$

where $\epsilon_{k}^{0}$ is the bare band dispersion, and $\Sigma(\mathbf{k}, \omega)$ is the self-energy.

We note that we neglect the instrumental resolution here since the feature of interest is large compared to the resolution. In the following, we will assume weak momentum dependence of this extracted self-energy (i.e. $\Sigma(\mathbf{k}, \omega) \rightarrow \Sigma(\omega)$.

Fig. 6 shows the comparison of the raw ARPES data (first row, Fig. 6a-c) and the parameterized data (second row, Fig. 6d-e). We parameterize the ARPES data with the form given by Eq. A1 where the spectral function $\mathcal{A}(k, \omega)$ is given by Eq. A2. In the fitting, we do not assume any form of the self-energy and the matrix element (i.e. every point of the fitted self-energy or matrix element is a free parameter in the fitting procedure.) The bare dispersion used here is the simple form given by the tight-binding (TB) parameters which is fitted to the LDA calculation shown in Fig. 1f and 2. The bare dispersion is given by $E(k)=-2 t\left[\cos \left(k_{x} a\right)+\cos \left(k_{y} b\right)\right]-$ $2 t^{\prime} \cos \left(k_{x} a\right) \cos \left(k_{y} b\right)-2 t^{\prime \prime}\left[\cos \left(2 k_{x} a\right)+\cos \left(2 k_{y} b\right)\right]-E_{F}$ where the TB parameters are $t=0.435, t^{\prime}=-0.1, t^{\prime \prime}=$ 0.038 , and $E_{F}=-0.5231 \mathrm{eV}$. For simplicity, we use constant background in the fitting procedure.

The fitting of the ARPES data (Fig. 6d-e) is very well in agreement with average error $<4 \%$. Since the extracted self-energy does not show a strong momentum dependence, the approximate 3 -dimensional spectra may be generated from this information as shown in Fig. 6 i.
* non@stanford.edu

$\dagger$ zxshen@stanford.edu

1 Z.-X. Shen et al., Phys. Rev. Lett., 70, 1553 (1993).

2 A. Damascelli, Z. Hussain, and Z.-X. Shen, Rev. Mod. Phys. 75, 473 (2003).

3 P. V. Bogdanov et al., Phys. Rev. Lett. 85, 2581 (2000).

4 A. Kaminski et al., Phys. Rev. Lett. 86, 1070 (2001).

${ }^{5}$ P.D. Johnson et al., Phys. Rev. Lett. 87, 177007 (2001).

6 A. Lanzara et al., Nature 412, 510 (2001).

7 X. J. Zhou et al., Nature 423, 398 (2003).

8 T. Cuk et al., Phys. Rev. Lett. 93, 117003 (2004).

${ }^{9}$ X. J. Zhou et al., Phys. Rev. Lett. 95, 117001 (2005).

10 W. Meevasana et al., Phys. Rev. Lett. 96, 157003 (2006).

11 M. C. Schabel et al., Phys. Rev. B 57, 6090 (1998).

12 R. Hayn et al., Phys. Rev. B 60, 645 (1999).

13 H. Lin, S. Sahrakorpi, R. S. Markiewicz, and A. Bansil, Phys. Rev. Lett. 96, 097001 (2006).

${ }^{14}$ Y. Chen et al., Phys. Rev. Lett. 97, 236401 (2006).

15 S. Sahrakorpi, M. Lindroos, R. S. Markiewicz, and A. Bansil, Phys. Rev. Lett. 95, 157601(2005).

16 T. Greber, T. J. Kreutz, and J. Osterwalder, Phys. Rev.
Lett. 79, 4465 (1997).

17 In examining various published band structures of Bi2201 and Bi2212 (see, e.g. references cited below), we find that the LDA in all cases gives a lower binding energy for band $\mathrm{B}$ than the experimental value. V. Bellini et al., Phys. Rev. B 69, 184508 (2004); D. J. Singh and W. E. Pickett, Phys. Rev. B 51, 3128 (1995); M. S. Hybertsen and L. F. Mattheiss, Phys. Rev. Lett. 60, 1661 (1988); P.A. Sterne and C.S. Wang, J.of Phys. C (Solid State Physics) 21, L949 (1988).

18 F. Ronning et al., Phys. Rev. B. 71, 094518 (2005).

19 D. van der Marel et al., Nature 425, 271 (2003).

20 M. R. Norman and A. V. Chubukov, Phys. Rev. B. 73, 140501(R) (2006).

21 N. Mannella et al., Nature 438, 474 (2005).

${ }^{22}$ K. M. Shen et al., Phys. Rev. Lett. 93, 267002 (2004).

23 E. Dagotto, A. Nazarenko and M. Boninsegni, Phys. Rev. Lett. 73, 728 (1994).

24 A. Macridin, B. Moritz, T. Devereaux and M. Jarell, private communication

25 T. Yoshida et al., Phys. Rev. Lett. 95, 146404 (2005). 

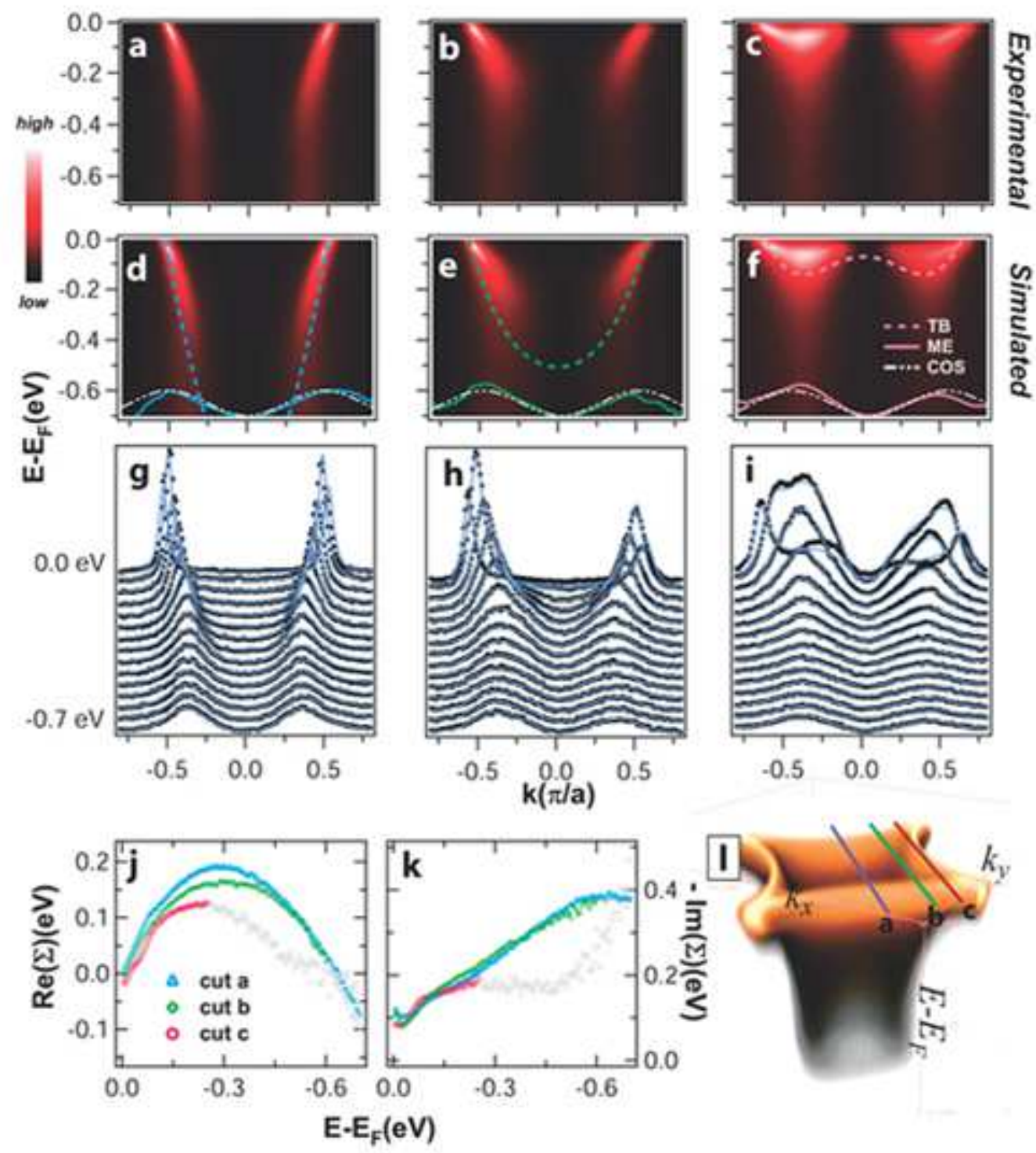

FIG. 6: On the first row, a)-c) are the experimental ARPES data along momentum direction as indicated by the band shown in bottom right. On the second row, d)-f) are the corresponding simulated images from the $2 \mathrm{D}$ analysis. The thicker color dashed lines are the dispersions generated from the tight-binding (TB) parameters given above. The solid color lines are the matrix elements (ME) obtained from the $2 \mathrm{D}$ analysis where the smaller white dashed lines are the empirically guessed form of the matrix element in the form of a cosine function (COS). On the third row, g)-i) are the MDCs of raw data (black dots) and corresponding simulated images (blue line). j) and k) are the extracted real and imaginary parts of the so-called self-energy in Eq. A2 for the cuts a, b and c; the extracted values are plotted in colors up to the energy not far from the bottom of the bare band (up to $0.6 \mathrm{eV}$ for cut $\mathrm{b}$ and $0.25 \mathrm{eV}$ for cut $\mathrm{c}$ ) and in grey at higher energy. l) shows the band structure generated from the TB parameters and the loosely-called self-energy along the nodal direction.

26 I.A. Nekrasov et al., Phys. Rev. B 73, 155112 (2006).

27 J. K. Freericks, T. P. Devereaux, R. Bulla, and Th. Pruschke, Phys. Rev. B 67, 155102 (2003).

28 S. Uchida et al., Phys. Rev. B 43, 7942 (1991).

29 S. Sugai et al., Phys. Rev. B 68, 184504 (2003).

30 N. W. Ashcroft and Mermin, N.D. Solid State Physics (Harcourt, Inc., Orlando, 1976), Chap. 17, pp. 334-337.
31 In the long precess of preparing this manuscript, we became aware of other experiments by J. Graf et al., cond-mat/0607319; B. P. Xie et al., cond-mat/0607450, T. Valla et al., cond-mat/0610249, Z.-H. Pan et al., cond-mat/0610442; J. Chang et al., cond-mat/0610880 